\title{
High-fat diet is associated with endotoxemia and low-grade inflammation
}

\author{
Shrestha UK ${ }^{1^{*}}$ \\ ${ }^{1}$ Department of Medicine, Manipal College of Medical Sciences, Pokhara, Nepal
}

*Corresponding Author:

Dr. Umid Kumar Shrestha, MD, PhD

Department of Medicine

Manipal College of Medical Sciences

\& Manipal Teaching Hospital

Pokhara, Nepal

Email: umidshrestha@gmail.com

\section{Citation}

Shrestha UK. High-fat diet is associated with endotoxemia and low-grade inflammation. Nepal Journal of Medical Sciences. 2012;1(2): 62-3.
Endotoxin (lipopolysaccharide [LPS]) is located at the outer membrane of the Gram-negative bacteria such as Escherichiacoli, Salmonella, Shigella, Pseudomonas, Neisseria, Haemophilus influenzae, Bordetella pertussis and Vibrio cholerae, and is regarded as one of the potent virulence factors of these bacterial species. In the circulation system, $80-97 \%$ of it is bound to the lipoproteins. The subclinical elevation in circulating endotoxin level is known as the metabolic endotoxemia. This elevation is not overtly apparent in a clinical setting but is being investigated as a significant potential etiology for several chronic diseases. The effect of high doses of LPS (10-200 ng/ml) on inflammation is well known, but the research is still going on about the effect of very low doses of LPS (1-100 pg/ml) on inflammatory and metabolic alterations.

A small but significant increase in pro-inflammatory cytokines and chemokines may occur with a persistent exposure to very low doses of LPS. Thus, this metabolic endotoxemia may lead to the chronicity of low-grade inflammation observed in many of the disease pathologies including type 2 diabetes mellitus, atherosclerosis, Parkinson's disease and cancer metastasis. This model may explain the development of low-grade, chronic inflammation, at least in part due to the lack of an appropriate counter response through the inadequate expression of antiinflammatory mediators and negative feedback loops. ${ }^{1}$

One of the prominent and emerging sources of metabolic endotoxemia is the consumption of high-fat diets, which may facilitate the gut absorption of endotoxin and change the gut flora that allows the endotoxin to enter systemic circulation..$^{2-5}$ It may be possible that the altered gut populations affect the amount of lipid absorbed by the gut, therefore allowing greater amounts of LPS into circulation. Moreover, the risk of LPS "leaking" into the bloodstream may also be increased by the differences in gut bacterial population dynamics and the general increase in bacterial load.

One recent study showed that just eating a high-fat "Westernstyle" diet for one month produced a dramatic $71 \%$ increase in 
plasma levels of endotoxin activity in comparison to a "prudentstyle" diet. ${ }^{6}$ This study revealed that the Western-style diet might contribute to endotoxemia by altering the gastrointestinal barrier function or the composition of the microbiota. ${ }^{6}$ The effect of highfat diet on metabolic endotoxemia seems to be reversible by the study, but one question arises. Could the development of metabolic endotoxemia by high-fat diet be prevented by antibiotics or probiotics? In fact, the therapeutic approaches aimed at reducing the translocation of endotoxin from the gut to the circulation need to be explored with regard to the possible prevention of several chronic diseases, which occur in the context of the chronic lowgrade inflammation.

Keywords: High-fat diet; metabolic endotoxemia; low-grade inflammation

References:

1. Chang S, Li L. Metabolic Endotoxemia: A Novel Concept in Chronic Disease Pathology. J Med Sci 2011;31:191-209.

2. Cani PD, Amar J, Iglesias MA, et al. Metabolic endotoxemia initiates obesity and insulin resistance. Diabetes 2007;56:176172.

3. Cani PD, Neyrinck AM, Fava F. Selective increases of bifidobacteria in gut microflora improve high-fat-diet-induced diabetes in mice through a mechanism associated with endotoxaemia. Diabetologia 2007;50:2374-83.

4. Manco M, Putignani L, Bottazzo GF. Gut microbiota, lipopolysaccharides, and innate immunity in the pathogenesis of obesity and cardiovascular risk. Endocr Rev 2010;31:81744.

5. Amar J, Burcelin R, Ruidavets JB, et al. Energy intake is associated with endotoxemia in apparently healthy men. Am J Clin Nutr 2008;87:1219-23.

6. Pendyala S, Walker JM, Holt PR. A high-fat diet is associated with endotoxemia that originates from the gut. Gastroenterology 2012;142:1100-1. 